Rev. Elev. Méd. Vét. Pays trop., 1966, 19, I (45-62).

\title{
Etudes Pastorales en Adamaoua Camerounais
}

\author{
par J. PIOT
}

\begin{abstract}
RÉSUMÉ
Le plajeau de l'Adamaoua par ses conditions climatiques et sanitaires favorables est une région d'élevage extensif en voie de surcharge; les principales raisons de la surcharge sonf l'augmentation globale du cheptel d'une part et d'autre part l'abandon de zones pastorales épuisées par un parcours abusif ou réputées infestées par les glossines.

If est donc envisagé ici d'étudier l'évolution des pâturages de base sous l'action de certains modes d'exploitation. Des remèdes ou techniques d'amélioration sont proposés dans certains cas.

Enfin, il est proposé un mode d'exploilation basé sur la rotation des pâtures pour la saison des pluies et pour la saison sèche.
\end{abstract}

\section{I. - CONDITIONS NATURELLES}

L'Adamaoua au sens large se présente comme un plateau élevé ( 900 à $1.800 \mathrm{~m}$ moyenne $1.100 \mathrm{~m}$ ) orienté sensiblement Est-Ouest sur 900 à $1.000 \mathrm{~km}$ entre les $6^{\circ}$ et $8^{\circ}$ de latitude Nord. II couvre pour le Cameroun environ $100.000 \mathrm{~km}^{2}$ dont une vingtaine de mille pour le Cameroun Occidental. Ce plateau décrit un arc de cercle à concavité ouverte vers le Sud; il présente une bordure Nord ef surtout Nord-Ouest assez élevée cependant que l'altitude générale décroît mollement vers le Sud. La retombée Nord, sur les bassins du Tchad ou du Niger est par contre relativement brutale et explique en partie les fortes précipitations reçues par ce front Nord du plateau.

Le climat est bien sûr de type tropicai, classé comme soudano-guinéen d'altitude. La pluviométrie est élevée se tenant aux alentours de $1.600 \mathrm{~m} / \mathrm{m}$ pour Ngaoundéré mals avec les amplitudes mensuelles et annuelles considérables que I'on trouve toujours en Afrique dans les zones à saisons sèches marquées (Tableau l). Cette saison sèche porte sur 4 à 5 mols écologiquement secs avec des minima d'humidité relative très bas ( 15 p. 100 en février). Ce caractère relativement rude de la saison sèche doit demeurer présent à l'esprit chaque fois que l'on envisage des introductions d'espèces car le chiffre total des précipitations n'est qu'un des éléments du choix des espèces; il est hélas souvent prépondérant parce que seul à être suffisamment bien connu.

La température très modérée a une moyenne annuelle de $22^{\circ}$ avec maximum absolu de l'ordre de $35^{\circ}$ (en mars) et minimum absalu aux alentours de $10^{\circ}$ (en janvier). Mars est le mois le plus chaud avec 2403 de moyenne et août le plus froid avec 2105.

L'indice d'aridité de De Martonne égal à 50 indique le «règne exclusif de la forêt» qui pourrait fort bien en effet avoir été la formation recouvrant l'Adamaoua dans un lointain passé.

La géologie de l'Adamaova est extrêmement marquée par de multiples manifestations de volcanısme qui s'échelonnent depuis le Crétacé moyen jusqu'à une époque récente, historique en tous cas (voire actuelle). Si donc le sous-sol est surtout formé de granites, gneiss ef migmatites (très schématiquement) les sols eux sont essentiellement d'origine volcanique ou marqués (dans leurs types d'argile par exemple) par des 
TABLEAD Na I

Relevés pluviométrıques de N'Gaourdéré *

\begin{tabular}{|c|c|c|c|c|c|}
\hline Mois & $\begin{array}{l}\text { Moyennes de } \\
34 \text { ans }\end{array}$ & $\begin{array}{l}\text { Nbre de } \\
\text { jours }\end{array}$ & $\begin{array}{l}\text { Max, en } \\
24 \mathrm{H} \text {. }\end{array}$ & $\begin{array}{l}\text { Mnnimum de } \\
\text { précipitation }\end{array}$ & $\begin{array}{l}\text { Maximum de } \\
\text { précipitation }\end{array}$ \\
\hline $\mathrm{J}$ & 3,8 & 0 & 42 & 0 & 42,0 \\
\hline $\mathbf{F}$ & 1,2 & 0 & 19,5 & 0 & 19,5 \\
\hline $\mathbf{M}$ & 42,8 & 5 & 52,5 & 0 & 159,7 \\
\hline A & 146,1 & 13 & 70,9 & 48,3 & 324,0 \\
\hline$M$ & 205,6 & 18 & 79,2 & 77,5 & 345,0 \\
\hline $\mathrm{J}$ & 240,8 & 20 & 104 & 144,9 & 488,5 \\
\hline $\mathrm{J}$ & 270,7 & 20 & 115 & 147.8 & 570,6 \\
\hline A & 274,2 & 20 & 100,5 & $\$ 14,9$ & 429,5 \\
\hline $\mathrm{S}$ & 242,4 & 20 & 80 & 140,5 & 492,2 \\
\hline 0 & 160,5 & 14 & 48,5 & 66,1 & 299,0 \\
\hline $\mathbb{N}$ & 12,3 & 1 & 50,1 & 0 & 55,6 \\
\hline$D$ & 3,4 & 0 & 54 & 0 & 54 \\
\hline ARUUEL & 1603,8 & 131 & & 1217,0 & 2229,4 \\
\hline
\end{tabular}

* établis sur 34 années

recouvrements volcaniques aujourd'hui disparus. Par ordre d'importance décroissante nous trouvons donc:

- des sols d'origıne volcanique (basaltique surtout),

- des sols d'origıne granitique,

- des sols sur gneiss ef migmatites,

- des sols de type hydromorphe,

- enfin des sols sur grés.

Malgré un pouvoir de rétention pour l'eau très faible pour certains sols (sols d'origine granitique par exemple) l'eau est partout présente dans l'Adamaoua. Aucun point n'est en saison sèche à plus de $5 \mathrm{~km}$ d'un point d'eau et l'abreuvement des troupeaux ne sera jamais ici un facteur limitant pour l'élevage.

Sous de telles conditions naturelles la végétation est assez homogène sur tout le plateau ; la formation la plus courante est la savane boisée à Lophrra et Daniellia dans l'étage domınant et Hyparrhenia Sp. plur. dans le tapis herbacé.
Tout l'ensemble est largement pénétré par un réseau tortueux de galeries forestières étroites auxquelles sont restés accrochés parfois des lambeaux de forêts denses, vestiges incontestables du passé. Nous avons pu voir disparaître en 2 ans I'un de ces massifs de $400 \mathrm{~m}$ de large sur $3 \mathrm{~km}$ de long sous l'action des défricheurs aidés du feu.

Les galeries subissent le même traitement, ravagées par la hache ef le feu, cultivées 1 ou 2 ans en mais sans techniques conservatrices de fertilité puls abandonnées et livrées aux intrusions des feux de savane qui les achèvent. Le maïs est souvent transformé en boisson et l'on a pu dire de ces défricheurs qu'ils «buvaient les galeries $\gg$.

Le passé historique (invasion des éleveurs Foulbés il y a environ 150 ans), les conditions naturelles favorables (rareté de la trypanosomiase, présence de galeries, donc d'eau, partout, tapis herbacé riche, etc...) ont fait de ce pays 
une terre d'élevage privilégiée. Malgré la vocation agricole incontestable d'une grande partie de l'Adamaoua, l'élevage extensif itinérant, voire vagabond, sera encore pour longtemps un état de fait que la faible densité de population (environ $3 \mathrm{hab} .\left(\mathrm{km}^{2}\right.$ ) ne pourra faire évoluer que lentement.

\section{II. - QUELQUES ASPECTS DYNAMIQUES DES PÂTURAGES DE L'AMADAOUA.}

\section{A) Pâturages de base.}

Nous avons dit de la savane qu'elle était du type à Hyporrhenia et effectivement il est rare que la somme des surfaces de base couvertes des différentes espèces d'Hyparrhenia ne soif pas dominante dans les relevés.

En l'absence de pâturage de saison des pluies, mais parcours de saison sèche avec feux précoces, les sols à évolution ferrallitique assez marquée sont couverts d'un tapis du type no 1 du tableau II.

Ce relevé est caractérisé par le très faible taux de Panicum phragmitoides d'une part et surtout par la présence à un taux relativement élevé de Loudetia orundinacea qui disparaît à peu près complètement des sols régulièrement pâturés en saison des pluies. Sur les sols suffisamment évoluès et profonds et dont la rétention pour l'eau est assez élevée l'Andropogon goyanus devient un élément jamaıs très abondant $(10 \mathrm{p}$. 100 d̀ 15 p. 100) mais très caractéristique du faciès graminéen ; avec ses longues feullies à faux pétioles et sa teinte vert glauque il se remarque particulièrement bien dans le tapis herbacé en toute saison.

Sur les sols jeunes à pente élevée et faible rétention pour l'eau (sols sur socle granitique, sols sur édifices volcaniques récents, etc...) dans les mêmes conditions de parcours, les analyses sont du type (2). Dans ce type de relevé les éléments physionomiques marquants sont Andrapogon schirensis et surtout Loudetia kagerensis dont l'aspect particulier attire l'œil.

On peut sans aucun doute dans ces types (le premier surtout qui est le plus répandu et recouvre une plus grande variété de sols) retrouver les marques écologiques et fabriquer des associations plus ou moins caractéristiques. En fait là, comme en foresterie, nous sommes plus sensıbles à la notion de formation que d'association dès que l'un des facteurs d'évolution intervient d'une façon prépondérante. Ici, le traitement imposé aux pâturages, leur mode d'exploitation sont plus déterminants que l'association de base dans l'évolution spécifique des terrains de parcours. C'est pourquol nous n'insisterons pas sur les sous-types écologiques dans lesquels telle ou telle espèce est favorisée par rapport aux autres. Les faciès à Hyparmenia rufa plutôt que diplandra ou que filipendula ou bracteata évolueront de la même façon à peu de chose près; de même les types caractérisés par Brachiaria brizantha ou Setario sphacelato ou Pennisetum purpureum, etc. auront des stades ultimes d'évolution identiques aux précédents.

Les associations liées à des conditions extrêmes resteront sous point d'interrogation parce que précisément le traitement qu'ils subissent est anormal. Nous pensons là aux pâturages. sur terrains inondés temporairement en salson des pluies ou à ceux des cuirasses à Andropogon sp. plur., Loudetia sp. plur., Ctenium newtonn, etc... jamais parcourus ou seulement en saison sèche.

\section{B) Méthode traditionnelle foulbé d'utilisation des pâturages.}

Les éleveurs Foulbés ef Bororos appliquent un mode d'exploitation des pâturages propre d̀ conserver le type à Hyporrhenia du relevé no 3 par exemple. Andropogon goyonus a tendance à diminuer, ce qui s'explique par sa très grande appétabilité, ef c'est avec la disparition de Loudetio arundinacea le meilleur indicateur de ce type de parcours. Avec un pourcentage marqué d'Andropogon schirensis, les pâturages à Loudetio kagerensis peuvent être ramenés à ce type avec moindre importance du taux d'Hyporrhenia: nous n'insisterons pas sur la difficulté qu'il y a à faire parcourir ces formations à des zébus trop grégaires et « glaneurs » de tempérament alors que des animaux comme les N'Damas plus individualistes et laborieux au pâturage sont capables en quelques années de se «fabriquer » un pâturage correct dans une savane à Laudetia pourtant peu aftırante.

Mais nous avons à préciser ce qu'est le pâturoge traditionnel.

Becucoup moins anarchique qu'il semble à première vue la technique, en saison des pluies, consiste à faire pâturer une zone bıen détermi- 


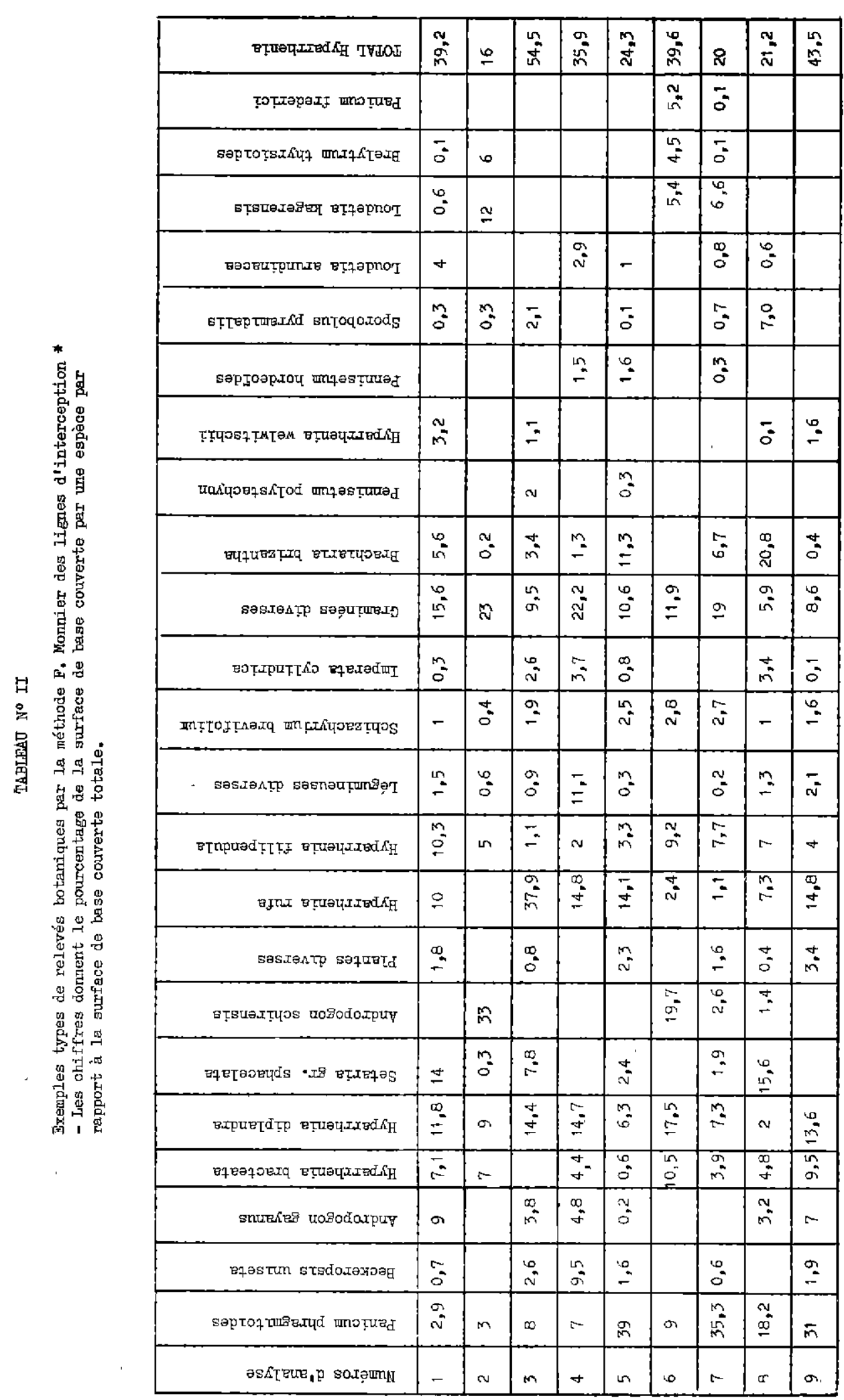


née 2, 3 à 6 ans (rarement plus) puis à l'abandonner complètement 3 à 4 ans pour. aller s'installer ailleurs. Les zones parcourves sont soumises ò un passage fréquent des troupeaux ce qui donne à l'herbe un temps de repos très court (10 à̀ 15 jours ou même moins).

La saison sèche est abordée avec pas mal de refus si le troupeau est trop léger ou bien avec un tapis bien ras si la charge a été suffisante. L'abandon est décidé lorsque le pâturage présente des signes d'appauvrissement (baisse de vigueur des Hyparrhenio, envahissement d'espèces non fourragères comme nous le verrons plus loin) et d'envohissement ligneux. Alors intervient la mise en repos avec passage de feux violents offensifs pour la végétation arbustive. Le parcours de salson sèche est effectué après feu sur tous les terrains disponibles, tant Il est vrai que la baisse de productivité de l'herbe impose l'exploitation de surfaces accrues (4 ou 5 fois plus, traditıonnellement, grâce à la transhumance).

Pendant le repos du paturage, le réensemencement en graminées pérennes est réalisé dès la première année en général (même lorsqu'il semble que plus rien ne fructifie); puis en 2 ou 3 ans des souches vigoureuses se sont reconstituées et le parcours peut être repris.

\section{C) Types de surcharge.}

La définition même du terme surcharge mériterait un développement que la suite de cet exposé nous évitera. Ce sera pour nous une charge telle qu'associée à certain mode d'exploitation du pâturage elle en amène la dégradation sous des formes diverses.

10 Surcharge violente. Elle nait du maintien en permanence sur un terrain d'une quantité excessive de bétail. Toute l'herbe est broutée ef rebroutée au fur et à mesure de sa repousse. La végétation arbustive est énergiquement rabattue par la dent et le piétinement incessant du bétail qui en outre effrite le sol et l'expose à une érosion intense. Le feu est tout de même allumé à peu près partout; le maigre regain, les arbustes sont visités et le sol encore émietté se trouve tout disposé à disparaître aux premières pluies laissant çà et là quelques touffes proéminentes de graminées pérennes cespiteuses qui se raréfient d'année en année. En 2 à 3 ans, l'herbe a disparu comme le sol d'ailleurs sauf quelques petites plages de Sida, Cassia tora et composées herbacées.

Ce tableau sombre ne se réalise généralement que sur des zones restreintes de rassemblement des troupeaux (Lahorés, pistes à bétail, marchés dispensaires, etc...) et a de ce fait un caractère quelque peu artificiel. Cependant des exemples de vastes zones ainsi dénudées existent (région de Banyo par exemple) formées dans la dernière décade qui a dô voir le rassemblement d'une masse considérable de bétail.

$2^{\circ}$ Surcharge prolongée. Nous pouvons considérer que ce paragraphe complète la partie qui traite de la méthode traditionnelle d'exploitation des pâturages. En effet, alors que les signes d'épuisement devraient commander la mise en repos jadis respectée, le parcours continue à s'effectuer et l'herbe constamment rabattue n'arrive plus à reconstituer ses réserves.

Dans ces conditions, l'évolution de la végétation est du type suivant.

a) Végétation herbocée graminéenne. Dans le premier temps décrit de diminution de vigueur des touffes celles-ci semblent cependant s'étaler, se plaquer au sol probablement par le piétinement ef l'augmentation de l'éclairement au niveau du sol qui favorisent le tállage. Il en résulte pendant cette période la constitution d'une sorte de pelouse fermée où les grandes graminées (Ponicum phrogmitoïdes, 'Huparthenia sp. plur., Andropogon type goyonus ef Andropogon schirensis, etc...) ont totalement perdu leur aspect classıque ; elles le retrouvent en partie à l'épọque de la fructification en réussissant des inflorescences à peu près normales sur des chaumes plus grêles et plus courts en rapport avec l'importance des souches.

Puis intervient typiquement la phase de dégradation des espèces pérennes par éclatement des souches d̀ partir du centre (description faite dans African Soil-C. C. T. A. vol. VIII, $n^{0} 2$ ) ; la couverture du sol diminue à nouveau et est progressivement assurée par des espèces gramınéennes annuelles (Paspalum scrobiculatum, Sporobolus molleri, patulus tenuis, granularis, Digifaria ternata, Pennisetum hordeoides, etc...) puis par des plantes diverses plus ou moins rudérales et nitratophiles (Triplotoxis, Sida, Chrysonthellum, Aconthospermum, labiées diverses, Sibthorpio europaea, Nephrolepis au pied des cepées, etc...). 


\section{b) Végétation ligneuse.}

L'évolution importante dans ce cas de charge concerne incontestablement la végétation ligneuse. Les premiers résultats d'étude de l'évolution de la végétation ligneuse entrepris à la Station Fourragère de Wakwa semblent bien indiquer qu'une charge élevée de bétail (500 kg/ha en début de saison des pluies, 650 environ à la fin) est propre à entraîner une diminution du peuplement arbustif plus importante même que le feu, qu'il soit tardif ou non. Mais en fait, dans le type de surcharge que nous examinons l'appauvrissement progressif du tapis graminéen est tel que la charge passe de $500 \mathrm{~kg}$ ou plus les premıères saisons des pluies à 100 ou $150 \mathrm{~kg}$ par ha. Les feux de saison sèche passent certes, mais sans endommager les arbustes ef sans entraîner la formation d'un regain (souches épuisées) qui pourrait susciter un parcours quelque peu important. On assiste alors à une reprise très vigoureuse du terrain par la végétation ligneuse. Les espèces les plus sensibles au feu apparaissent comme :

Harungana madagascariensis,
Maesa sp.,
Vernonia sp. plur. (dont amygdalina et
ngaounderensis),
Fagara welwitschii,
Croton macrostachyus,
Steganotaenio araliacea,
Phyllonthus muellerianus,
Ekebergia senegalensis.

En outre par endroits se répandent des espèces comme Nauclea latifolia, Mussaenda sp. etc... ou encore propagées en abondance par le bétail (graines dans les déjections) des espèces comme Piliostigma thonningir.

Une ambiance nouvelle s'installe mais en ce qui concerne les pâturages, leur surface exploitable diminue considérablement par suite de l'augmentation en nombre et en taille des cepées d'une part et du nombre de leurs tiges qui grignotent par leur couvert les plages pâturables.

Enfin le phénomène se complète par une destruction du sol due au piétinement qui doit de plus en plus se farre électivement entre les cépées.

Une fois le processus de reprise par la forêt entamé, il y a irréversıbılıté naturelle. Seul un défrichement pourrait I'enrayer mais ne serait certainement pas souhaitable car le tapis graminéen épuisé est absolument incapable d'assurer la relève en ce qui concerne le couvert du sol et sa protection.

Le débroussaillement sélectif d'abord (espèces non appétées) ne doit être envisagé qu'oprès une mise en defens absolve d'environ 3 ans sans feu sauf s'il peut être assez violent.

L'évolution naturelle de ces terrains se fera dans le sens d'une savane arborée dense allant jusqu'à la forêt claire (exemples dans la haute vallée du système de la $M$ 'béré) avec tapis herbacé court qui dans un stade plus avancé (une trentaine d'années semble-t-il) est essentjellement d base de Pennisetum hordeoides et Pennisetum pedicellatum.

II ne nous semble pas qu'à ce stade un équilibre soit atteint, car nous voyons mal comment est assurée la pérennité des espèces ligneuses; mais nous ignorons en combien de temps et comment exactement se fera le retour à la savane arborée à Hyparrhenia, Andropogon et Loudetia.

En définitive donc il s'agit là d'une véritable usure du pâturage due essentiellement à l'escamotage de la traditionnelle mise en repos. L'envahissement ligneux qui en résulte est sans doute un désastre pour l'éleveur mais convient fort bien au forestier dans ses fonctions de conservateur du sol car les dommages subis par ce dernier restent superficiels et momentanés. Dès que la végétation ligneuse a repris le dessus, la protection antiérosive est efficacement assurée. Peut-être un bon tapis graminéen seraitil plus efficace mais en tous cas pas après passage du feu (systématique et général d'ailleurs) qui livre aux premières pluies un sol nu vulnérable au «plash» des gouttes d'orages.

Nous termınerons ce paragraphe en soulignant longuement combien ce type d'évolution est grave en Adamaova et ce qu'il représente. Car se demandera-t-on pourquoi le parcours traditionnel n'est-il plus appliqué ? C'est que les terrains pâturables ne sont plus aujourd'hui disponibles et excédentaires comme à la «bonne époque »d'installation du nomadisme. Les raisons sont multiples et non indépendantes hélas.

Tout d'abord le simple fait démographique qui entraine une augmentation globale du cheptel ; cette augmentation est un phénomène séculaire non étranger à l'histoire des migrations 
peuhles. Mass maintenant, les déplacements vers l'Est et le Sud buttent sur des limites ; au Sud c'est la barrière de la trypanosomiase et sur l'axe Est-Ouest, les Etats voisins qui, subissant la même évolution, se saturent eux aussi s'ils ne le sont déjà. Partout on doit se contenter d'un domaine déterminé qui n'acceptera les accroissements numériques que si on le rend plus productif. C'est là le point de passage obligé vers des formes plus intensives d'exploitation.

Mais, à cette croissance s'ajoute un phénomène grave d'abandon de certaines zones par les éleveurs du fait de l'apparition récente, disentils, des trypanosomiases. Nous nous garderons bien d'exprimer une opinion au sujet du bienfondé de ces déclarations; mais nous devons conistater que la surcharge ne peut qu'être aggravée par cet état de fait, que les troupeaux tournent en rond sur leurs pâturages ou ceux des autres, qu'ils viennent de très lain traversant des' zones infestées de longue date cette fais, en entraînant la création de foyers nouveaux par transmission directe (d'où nouveaux abandons de pâturages). Cet aspect «boule de neige » des divers facteurs aboutıt pour l'instant à des surcharges de surfaces excessives. Souventencore la surcharge est du type que nous venons de décrire, mais aussi fréquemment, et dès que plusieurs éleveurs explaitent simultanément les mêmes terrains, nous nous trouvons devant le type de surcharge violente décrit précédemment qui débute par une régression de la strate arbustive ef se poursuit par la dégradation du sol et sa mise à nu avant que la végétatıon ligneuse se soit réinstallée. Le tout est de plus largement favorisé par l'installation et le déplacement fréquent des Waldés (campement de bergers où sont rassemblés pour la nuit, vaches et veaux surtout) qui constituent après abandon de véritables amorces de dégradation. Des exemples: de tous ces tableaux que nous venons de brosser existent bien sûr dans la région mais par-dessus tout, alors qu'on pensait à une lente évolution, on a dû constater que trois années pouvaient être suffisantes pour modifier profondément les pâturages.

3o Surchorge technique.

Cette dénomination un peu artificielle veut désigner un mode d'exploitation incapable d'assurer la pérennité spécifique des pôturages.
La charge globale n'est pas à proprement parler excessive et la formation reste fondamentalement pastorale, mais les espèces gramınéennes de valeur sont remplacées par d'autres fort médiocres.

Nous voulons traiter ici du mode de constitution des pâturages à Sporobolus pyramidalis appelé Pagamé par les Foulbés.

A l'origine, la charge doit être comparable à celle du type de surcharge précédent ef l'évoIution est identique surtout pour la végétation ligneuse qui disparaît. Mais ensuite, en 20 à 30 ans après passage par un stade à Pagamé, Hyparrhenia filipendula et Urelytrum thyrsiondes, on arrive à avoir un pâturage où la seule espèce de grande taille est le Sporobulus pyramidalis. Il se présente alors en grosses touffes cespiteuses de refus broutés à $40-50 \mathrm{~cm}$ de haut avec entre les touffes des espèces prostrées ou fines comme Digitaria minutiflora, Pospalum scrobiculatum, Setarı sphacelato variété petite.

Ces types de pâturages sont ceux créés par les éleveurs plus ou moins propriétaires de leurs pâturages et qui pour cette raison essaient de l'exploiter le plus rationnellement et le plus intensivement possible avec des troupeaux dont ils ont le contrôle sans craindre les perturbations causées par des troupeaux étrangers. Les caractéristiques du parcours sont:

- Existence d'une pseudo-rotation à temps de repos trop court.

- Pâturage permanent qui s'effectue tous les ans sans mise en repos de saison des pluies.

- Passage fréquent des animaux d'où résulte un piétinement excessif.

- Enrichissement important en déjections.

- Enfin passage systématique du feu en saison sèche.

Presque partout dans nos analyses botaniques le Sporobolus pyramidalis est présent à un faible taux de recouvrement lié au fait qu'ıl forme des brins isolés que les animaux peuvent brouter au ras du sol, il se trouve en fait souvent simplement étêté et jamais en tout cas en début de saison sûrement parce qu'il n'est pas excellent, maıs aussi parce qu'il fleurit très tôt dans la saison des pluies et que les sommités florales ne sont pas appétées. Donc, là où un pâturage sélectif 
peut s'exercer, les sporobolus (nous distınguons une variété de bas de pente à panicules étalées ef une variété de plateau à panicules fastigiées) qui ont le même comportement se trouvent délaissés et, lorsque la rareté de l'herbe oblige le bétail à y mettre la dent, il n'en peut consommer que le hout.

Les simples brins clairsemés du début, par piétinement et éclairement deviennent des touffes bien accrochées qui au stade final ont jusqu'à $20 \mathrm{~cm}$ de diamètre d̀ la base. Le sol n'est plus beaucoup couvert entre les touffes. mais les quelques gramınées basses qui s'y trouvent se maintiennent sans que le passage des troupeaux leur cause trop de dommage.

Compte tenu de la valeur alimentaire du sp. pyramidalss, ces pâturages ne valent plus guère que le $1 / 3$ ou la moltié de leur valeur d'origine. Néanmoins, ils sont sous cette forme très stable et le parcours continue à s'y exercer tous les ans avec une charge, légère par nécessité, qui a l'avantage d'éviter la dégradation du sal cependant qu'elle est suffisamment élevée pour empêcher une reprise arbustive dangereuse.

$4^{\circ}$ Amélioration des pâturages à Sporobolus pyramidalis.

Les surfaces couvertes par ces formations sont très importantes et posent un problème d'amélioration d'autant plus sérieux que, comme nous l'avons déjà dit elles concernent des élevages d'un type industriel plus intensif que d'ordinaire.

a) Passage à la déchaumeuse.

L'essal a été effectué à Goundjel $(80 \mathrm{~km}$ Est de Ngaoundéré) sur les terrains de parcours de la Compagnie Pastorale. L'extirpation est relativement aisée ef un passage croisé est suffisant. Mais la couverture de première année est faite d'espèces de jachère à base de graminées fines (Eragrostis, Chloris, Eleusine, Paspalum, etc...) et I'installation des andropogonées est lente. L'opération en est à so trossième saison des pluies seulement et est toujours suivie.

b) Mise en défens.

Un exemple de mise en défens a été observé à Jakiri (Cameroun Occidental vers Bamenda) où une parcelle d'un hectare a été délimitée et fauchée une fois par an pour fournir de la paille. Après 10 ans de ce traitement et sans feux, le retour des Hyporrhenia et la disparition des grosses touffes de Sporobolus étaient choses faites.

c) Enrichissement en Kikuyu (Pennisetum clandestinum).

Ces introductions se sont révélées particulièrement intéressantes dans la région de Bamenda et de Dschang (1.500 à $1.800 \mathrm{~m}$ d'altitude, 2-3 mais de saison sèche). Introduit sous forme de boutures ou d'éclat de souche, le Kikuyu stolonnise vigoureusement et occupe complètement les espaces entre touffes à la place des espèces habituelles.

Le Kikuyu n'élimine pas le pagamé, mais devient l'élément alimentaire de base du pâturage cependant que le pagamé permet d'améliorer le coefficient d'encombrement de la ration. Que devient la valeur exacte de ces pâturages? Nous espérons simplement pouvoir en faire l'estimation dans les années à venir mais elle correspond incontestablement à une amélioration très importante.

Cette méthode n'est malheureusement pas généralisable partout car la majeure partie de l'Adamaova a une saison sèche trop rude pour le Kikuyu alors que le risque d'envahissement du pagamé existe partout.

D) Des effets de la charge consiante ou de la charge insuffisante.

L'ensemble zootechnique de Wakwa s'est révélé être un champ d'étude remarquable pour ce genre d'exploitation. En effet, les exigences de sélection du centre zootechnique lui ont imposé de mettre un troupeau par parc sans faire de rotation que le prix élevé des clôtures interdisait. De même, de 1957 à. 1962 et avec analyses botaniques annuelles à. l'appui, des parcs de la Station Fourragère ont reçu un troupeau déterminé par parc du début à la fin de la saison des pluies. Les charges étaient réputées légères (200 d̀ $300 \mathrm{~kg} / \mathrm{ha}$ ) normales (300 à 450) ou excessives (450 à $550 \mathrm{~kg} / \mathrm{ha}$ ) et appliquées en fonction d'un protocole précis. La plupart des parcs avalent déjà été traités avant leur mise en expérimentation en sous-charge et leurs andyses de départ en sont déjà fortement affectées. L'analyse $n^{\circ} 4$ peut être un exemple de composition herbacée au départ (1957) et le no 5 pour le même parc 5 ans après. De même pour les analyses 6 et 7 respectivement. 
L'élément caractéristique de ces relevés est l'augmentation remarquable du Panicum phrogmitoides qui peut atteindre des taux de près de 60 p. 100. Cette importance de l'espèce est encore plus frappante à l'œil ; en fin de saison des pluies le Ponicum encombre le pâturage de ses refus d'autant plus hauts que la charge a été plus légère. Après une année de repos, mise à feu précoce puis charge assez élevée en rotation et sans feu pendant 2 ans, on a pu faire baisser le taux de Panicum de 10 à 20 p. 100, cependant que le taux des Hyparrhenia augmentait de 5 à 15 p. 100 dans les relevés. Pour nous ef partout dans l'Adamaoua, l'importance de la charge nous est révélée par le Ponicum qui est abondant dans les parties insuffisamment chargées depuis quelques années. Quant aux refus à la fin d'une saison des pluies, ils sont d'autant plus hauts que la charge, a été légère ou que l'intervalle entre 2 broutages successifs a été trop long au moins une fois au cours de la saison.

Ce développement du Panicum est assez logique car sans être franchement une mauvaise graminée il est délaissé par les zébus réputés pour faire de la pâture sélective. Ils ne consentiront à le consommer qu'après épuisement des autres espèces et si encore, à ce moment tà, il n'est pas devenu trop grand et trop dur. Le phénomène est à peu près le même pour Hyparrhenio diplandra qui est fort bien appété et peut fournir des pâturages courts ou au contraire d'énormes touffes de refus sur lesquelles les bêtes glanent les repousses axillaires tendres qui n'existent pas hélas sur Panicum. Quelques erreurs donc à éviter :

- une charge trop légère. Les bêtes peuvent se contenter des autres espèces :

- une charge constante qui permet un broutage sélectif par pâture d'une partie seulement du tapis herbacé au fur et à mesure de sa repousse ;

- un temps de passage en rotation trop long qui permet aux animaux un broutage répété (au cours du passage) des bonnes espèces aux dépens des autres moins appréciées ;

- un cycle de rotation trop long qui permet entre deux passages un trop grand développement de certaines espèces. Les bêtes sont prises de vitesse par la pousse de l'herbe;

- un cycle trop court ou un temps de repos trop court qui ne laissent pas à I'herbe une repousse suffisante et entraînent un épuisement des souches.

\section{III. - QUELQUES RÉSULTATS D'ESSAIS DE CHARGE}

\section{A) Saison des pluies}

10 Les données du problème.

Nous nous sommes trouvés à Wakwa en générali à la Station Fourragère en particulier avec des parcs où, au cours des ans on a «fabriqué » systématiquement du Panicum phragmitoïdes mal appété. Beaucoup de secteurs de l'Adamaoua sont eux aussi dans ces conditions et il était intéressant de savoir comment ces refus pouvaient être éliminés. Sans doute, avec des moyens mécaniques suffisants (cas de la Station Zootechnique dont 25 à 50 p. 100 des parcs d'environ 100 ha sont dessouchés et traités au girobroyeur 2 ou 3 fois par an) est-il possible de corriger et de limiter cette installation des, refus. Mais l'élevage extensif ne dispose pas de tels moyens eti nous pouvions voir suffisamment de pâturages d'éleveurs locaux bien conduits pour être persuadés qu'une technıque de parcours pouvait résoudre ce point.

Le seul critère de départ éłait physıonomique. Nous devions terminer la saison des pluies avec une pelouse courte, bien fermée où l'on n'aurait pas obligatoirement à mettre le feu ; ce dernier point nous permettrait de conserver un fourrage sur pied important en saison sèche.

\section{$2^{\circ}$ Les moyens.}

Disposant d'une trentaine de parcs de 3,5 à 12 ha bien connus depuis 7 ou 8 ans, un programme 1963 de charge en rotation a été éfabli. Sept séries de 3 à 6 parcs ont été constituées la charge variant de 300 à $500 \mathrm{~kg} / \mathrm{ha}$ (début de charge); les durées de charge variaient de 3 à 15 jours selon les parcs, les temps de repos de 8 a 37 jours ef la durée totale de la rotation de 19 à 40 jours. A la fin de chaque rotation, la pesée des troupeaux permettait un contrôle de la productivité. En 1964, 5 autres séries ont été suivies avec une meilleure approche des solu- 
tions dues à l'exploitation des résultats de l'année précédente.

\section{Les résultats.}

Les résultats ont été particulièrement démonstratifs; dans les mellieures conditions nous avons sur 4 parcs (sol sur basalte récent) obtenu du 30-4-63 au 28-11-63 un croît de 191,5 kg/ha soit une augmentation de 37,1 p. 100 du poids initial du troupeau (constıtué de zébus métıs FoulbésBrahman du Texas de $190 \mathrm{~kg}$ environ au début de l'opération).

La charge était en début de saison de $500 \mathrm{~kg} / \mathrm{ha} \mathrm{;}$ la rotation totale de 24 jours et les temps de passage sur les parcs de 4, 5, 6 ou 9 jours (fonction de la surface de chaque parc par rapport à I'ensemble). L'état du tapis herbacé était parfait en fin de saison (herbe basse et sol bien couvert) sauf un parc qui avait quelques touffes basses de refus de Panicum dues à un temps de passage trop élevé ( 9 jours). Les conclusions de ces divers essais sont les suivantes :

- temps de passage aussi bref que possible et en tous cas ne dépassant pas une semane :

- temps de repos de l'ordre de 3 semaines seulement ;

- pour l'Adamaoua, charge non inférieure à $500 \mathrm{~kg} / \mathrm{ha}$ et, fait surprenant, que le terrain soit granitique ou basaltique. On ne réussit pas à rabattre les refus à moins que cela ;

- enfin nous n'avons encore pas pu relever de différences significatives de productivité entre nos parcs dessouchés ef ceux qui ne le sont pas malgré un couvert ligneux parfols important.

Ce type de parcours, indépendamment de ses résultats globaux, nous a permıs d'éviter des pertes de poids de pleine saison (perfes mal expliquées mais associées aux périodes les plus pluvieuses et froides) et surtout de fin de saison des pluies. Cette perte bien avant la fin des pluies était l'une des conséquences de la charge constante créatrice de refus qui réduisent dans d'énormes proportions les parties de pâturages utilısables.

\section{B) Saison sèche}

C'est bien là que se situe le facteur limitant de l'élevage non pas par manque d'eau ici, mass par manque d'aliments.
Nous n'avons pas encore à présenter d'essals systématiques mals sommes en possession de données étayées par des pesées fréquentes de troupeaux complets.

Appliquant la règle bien connue du nombre d'ha égal au nombre de mois de saison sèche nous pensions que 5 ha pouvaient entretenir une bête donc 8 à 10 fois moins qu'en saison des pluies. E† effectivement nous sommes arrivés d̀ ces chiffres en terrain granitique brôlé à faible rétention pour l'eau. Après une mise en repos à peu près complète d'une saison des pluies nous avons brôlé le pâturage dont le boisement assez important est de 1.700 rejets, 900 arbustes et 40 arbres à I'ha. Un maigre regain est apparu qui a été consommé sur un ha par une bête de $400 \mathrm{~kg}$ en 6 jours puis une seconde fois deux mois après en 3 jours (en fait le terrain était utilisé par tout un troupeau). Dans ces conditions le poids carcasse se maintient à peu près. Ensuite, les animaux consommant les repousses ligneuses ont pu tenir 3 jours puis 1 jour sans trop de pertes semble-t-il. Les espèces ligneuses appétées représentent à peu près le $1 / 3$ de la végétation arborée et sont par ordre décroissant d'intérêt :

Daniellia oliver (folioles par folioles), Pllostigma thonningil (feuilles et gousses), Lophira lanceolata, Hymenocardia acida, Vitex cuneata ef madiensis, Gordenıa sp. plur., dont ternifolia, Bridelia ferruginea,

Ficus sp. plur., Ochna afzeli, Combretum sp., trichilia emetica, etc...

Sont absolument inutilisées :

Syzygium guineense var. macrocarpum, Terminalia macroptera et $T$. dewevrei, Protea elliotil,

Annona arenaria,

Psorospermum febrifugum,

Lannea kerstingu,

Entada, Albizia, etc...

En conclusion un ha de terrain granitique non dessouché porte une bête de $400 \mathrm{~kg}$ pendant 13 jours environ après quoi les bêtes perdent un poids considérable. Nous nous trouvons donc avec un besoin de 10 ha par animal de 
$300 \mathrm{~kg}$ (poids plus élevé que la moyenne) pour une saison sèche de 130 jours, ce qui n'empêchera pas le bétail de maigrir d'ailleurs.

Sur les terrains basaltiques et de bas fonds, la pousse de l'herbe est plus importante et surtout dure plus longtemps.

Deux groupes d'observations ont pu être effectués.

10 A la Station Fourragère début 1964, un troupeau de 56 mâles pesant 14 tonnes a passé la saison sèche sur quelques 150 ha non brûtés de parcours ordinaire où elles ont perdu environ 7 p.' 100 de poids en novembre et décembre. Sur parcelles d'essai à Stylosanthes gracilis, Brachioria brizantha, Digitoria «umfolozl», etc... couvrant environ 10 ha au total, les animaux ont repris 3,5 p. 100 de poids entre le 9 janvier et le 13 février puis nouvelle perte de poids de 2; 5 p. 100 en 1 mois stoppée par l'arrivée précoce des pluies. Donc malgré une superficie importante, sans les praıries artificielles on peut estımer que la perte de poids aurait atteint 10 à 12 p. 100 au cours d'une saison sèche pourtant courte.

En saison sèche 1965, sur parcs non brûlés basaltiques en majeure partie couvrant une centaine d'ha plus les parcelles d'essais déjà exploitées en 64 nous avons conservé un trovpequ de 19 t en 57 têtes.

Outre les prairies artificielles, les pâturages comportaient une bonne part de bas fonds (pourtour de marais) et malgré cela nous n'avons pas pu réussir cette fois une reprise de poids en cours de saison sèche. La perte de poids a été du début du mois de novembre au 19 mars de 13,5 p. 100 dont 10 p. 100 au 5 janvier. En falt Il semble bien que les animaux auraient pu perdre moins de poids s'ils avaient pu demeurer dans le même parc alors que notre cloisonnement nous, imposait un déplacement fréquent certainement préjudiciable à un bon porcours.

En tous cas, un mois $1 / 2$ après la réinstallation de la saıson des pluies (consıdérée comme acquise après un total de pluie de $50 \mathrm{~m} / \mathrm{m}$ ) tous les animaux ont récupéré leur polds maximum de la saison précédente et 15 jours à un mois après (suivant l'âge), la courbe normale de croissance est rattrapée ef le manque à gagner de salson sèche absorbé pour les animaux âgés au moins de 2 ans $1 / 2$.

20 A la Station Zootechnique de Wakwa pour la première fois des troupeaux complets ont passé toute la saison sèche sur des parcs bien déterminés. Le poids des animaux (pesés une fois tous les 2 mois) a pu être suivi et pour la première fors aussi on a pu empêcher la grosse majorité des pares de brûler.

Ces parcs sur terrains basaltıques possèdent à peu près tous quelques bas fonds, et couvrent une centaine d'ha dont à peu près la moitié est débarrassée de ses refus en fin de saison des pluies; l'autre moitié non dessouchée correspond à un tapis gramınéen allant du type $8 \mathrm{au}$ type 9 d'analyse. Pour ces dernières parties, la saison sèche est abordée avec présence de tout le Panicum sous forme de touffes de $40 \mathrm{~cm}$ à $1 \mathrm{~m}$ de haut et le terrain est donc sérieusement encombré. Les parcs de type 9 correspondent bien au type habituel à charge de saison des pluies insuffisantes alors que le type 8 représente un type plus évolué de pâturage où Setaria, Brachioria surtout ef Sporobolus indiquent; nous n'osons pas dire un début de dégradation, mats un parcours assez intense sans repos depuis de nombreuses années.

Au cours donc de la salson sèche, dans 6 parcs peu différents les uns des autres la perte a été partout d'environ 14 p. 100 pour des laitières d'à peu près $400 \mathrm{~kg}$ dont $1 / 3$ en iactation. Compte tenu du croit des veoux, la perte n'était plus que de 5 à 6 p. 100. Et cependant les charges à l'ha étaient très différentes et allaient de 110 à $250 \mathrm{~kg}$ à l'ha. Ces résultats sont réconfortants car avec une perte somme toute très limitée on peut tenir la scison sèche avec seulement 2,5 fois plus de pâturage qu'en saison des pluies. Il nous faut cependant nous souvenir que les parcs n'ont pas été incinérés et que les refus ont été fauchés faute de quoi nous aurions dû pratiquer la même transhumance que les autres années ce qui d'ailleurs n'empêchail pas les animaux de perdre aussi beaucoup de poids. Par conséquent, avec une charge de saison des pluies, propre à laisser un pâturage sans refus à l'entrée de la salson sèche, il nous suffira de chercher à caser un peu moins des $2 / 3$ du troupeau puisque nous passerons sur ces parcs d'une charge de 625 $650 \mathrm{~kg} / \mathrm{ha}$ à une charge d'environ $250 \mathrm{~kg}$. Ces terrains, nous aurons à les prendre dans des zones qui seront restées en repos et que nous devrons débarrasser des: grandes herbes non pas au girobroyeur «méthode de riche» mais hélas par le feu. Aurons-nous alors pour ces 
pâturages la même productivité qu'après une fauche et quel type de feu aurons-nous à mettre ? Nous espérons bien pouvorr y répondre dans l'avenir.

Enfin, nous avons pu encore suivre 3 parcs ayant une importante proportion de bas fonds et où les pertes de saison sèche onf été beaucoup plus réduites. En particulier un parc qui n'a perdu que 5 p. $100 \mathrm{malgré} u n e$ charge de $340 \mathrm{~kg} / \mathrm{ha}$ et un autre 6,6 p. 100 en moyenne avec $245 \mathrm{~kg} / \mathrm{ha}$ de génisses d'environ $250 \mathrm{~kg}$.

En conclusion et avec des lots mayens de pâturages sans transhumance, sı nous voulons effectuer un bon parcours de saison des pluies nous aurons à cette saison environ les $2 / 3$ de nos pâturages en repos, ou sı l'on préfère, la pâturage intensif de saison des pluies ne sera effectué qu'une année sur 3.

Et nous termınerons ce paragraphe en soulignant qu'il n'est pas du tout anormal que les pertes soient les mêmes pour des charges variant du simple au double; cela résulte simplement du fait que même avec un disponible d'aliments excédentaire, la forme sous laquelle ils se présentent ne permet pas aux animaux d'en récolter plus. Les animaux perdent une énergie énorme en déplacement et en mastication gaspillée pour des prises infimes.

Signalons que des pertes de saison sèche de 20 p. 100 et plus n'ont ici rien d'anormal et de désastreux sauf pour les animaux de moins de 18 mois.

\section{IV. - CONCLUSION}

Nous avons parlé de charges excessives, de charges insuffisantes et de charges donnant de bons résultats immédiats; mais ces dernières peuvent-elles être considérées comme correctes? En particulier peut-on considérer comme normal le fait que les mêmes charges soient appliquées sur des sols basaltiques riches ef des sols granitiques pauvres? II semble bien que non, et qu'il y a lieu de chercher quelque part une compensation. Ce facteur de correction, nous le cherchons dans la fréquence de mise en repos de saison des pluies des pâturages, mise en repos nécessaire pour permettre la reconstitution des souches, les réencemencements et peut-être, à l'occasion des feux, la limifation voir la régression du couvert arbustif. Nous ne ferons là rien de plus que ce qui était fait traditionnellement; nous essaierons seulement de le faire différemment préférant un aménagement annuel plus souple, plus adapté à la propriété de la terre qu'une exploitation basée sur des périodes de charge ou de repos de 3 à 6 ans probablement en partie liées au caractère nomade de l'élevage. En outre nous considérons que les risques de dégradation vialente du sol seront ainsi moins élevés. En tous cas nous ne voulons fabriquer ni du Sporobolus ni du Pancum à l'excès ni de la forêt. Nous travaillons sur des bases très proches des meilleures conditions d'exploitation extensive, ef l'expérimentation sera longue qui permettra qux différents signes d'évolution de se révéler; mals elle est nécessaire.

Elle est d'autant plus nécessaire qu'elle donnera des résultats utiles d̀ une exploitation intensive du bétail que nous préparons déjà et qui se dessine en maints endroits. En particulier, nous aurons alors à rendre plus compatibles les temps de rotation nécessaires à un broutage correct et les temps sûrement très différents donnés par' l'étude du meilleur temps de repos à laisser à l'herbe pour en obtenir le rendement fourrager maximum (matière sèche et protésne au moins).

Et nous terminerons par un retour sur l'aspect dynamique de la savane avec deux phrases d'Aubreville (climats, forêts et désertification de l'Afrique tropicale) :

«... l'emprise totale des savanes est l'œuvre de deux seuls fléaux, le défricheur et les feux... » auxquels 'l'éleveur (incendiaire mais peu défricheur) doit becucoup car une bonne partie de cette savane est son capital qu'il saura préserver nous l'espérons. Dans le cas contraire, espérons encore que la disparition des terrains de parcours se fera par «le pâturage abusif qui produit également des facies spéciaux de végétation forestière » qui sont peut-être ceux que nous avons signalés au cours de ces pages. Ce sera en tous cas un moindre mal.

N.D.L.R. - Les conclusions de l'auteur rejoignent celles de J. C. Bille, consignées dans son travail intitulé : «Pâturages du secteur occidental d'Elevage de la Républıque Centrafricaine $\gg$.

Instilut d'Elevage et de Médecine vétérinaire des Pays tropicaux.

Centre de Recherches Fourragères de Wokwa

(Comeroun) 


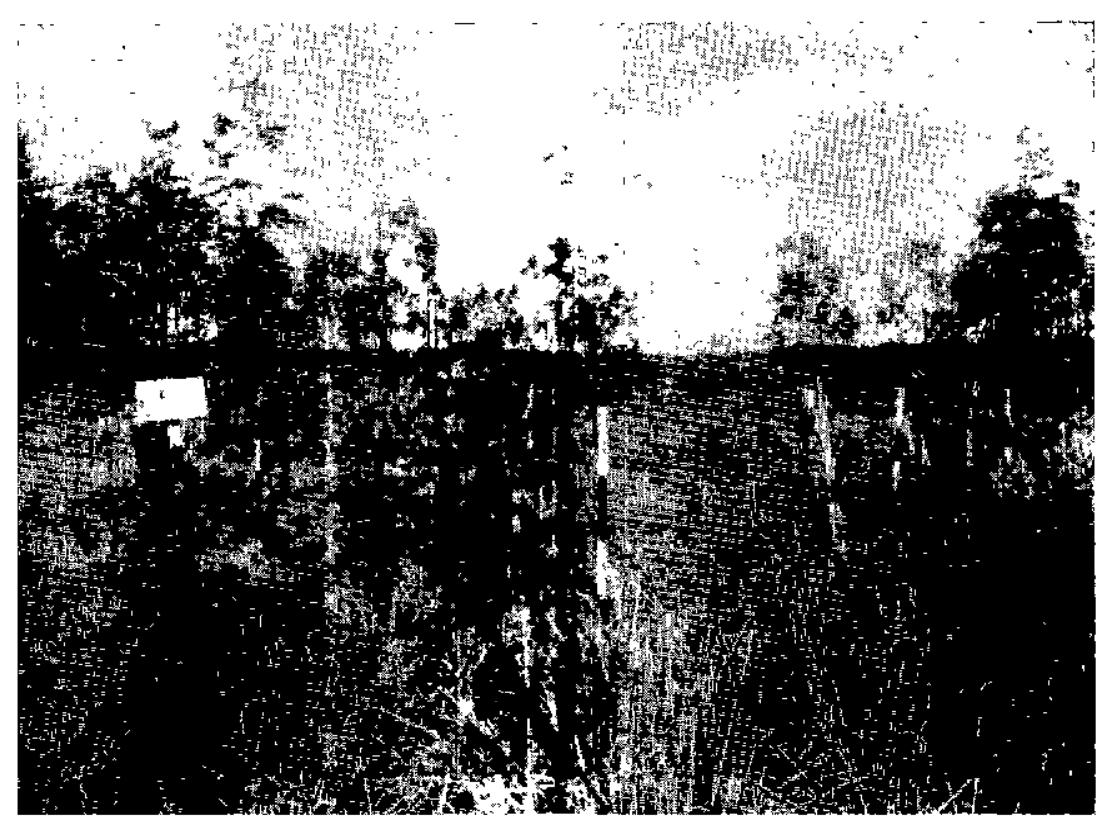

No 1. - Un groupe de 6 placeaux d'étude du meilleur temps de repos.

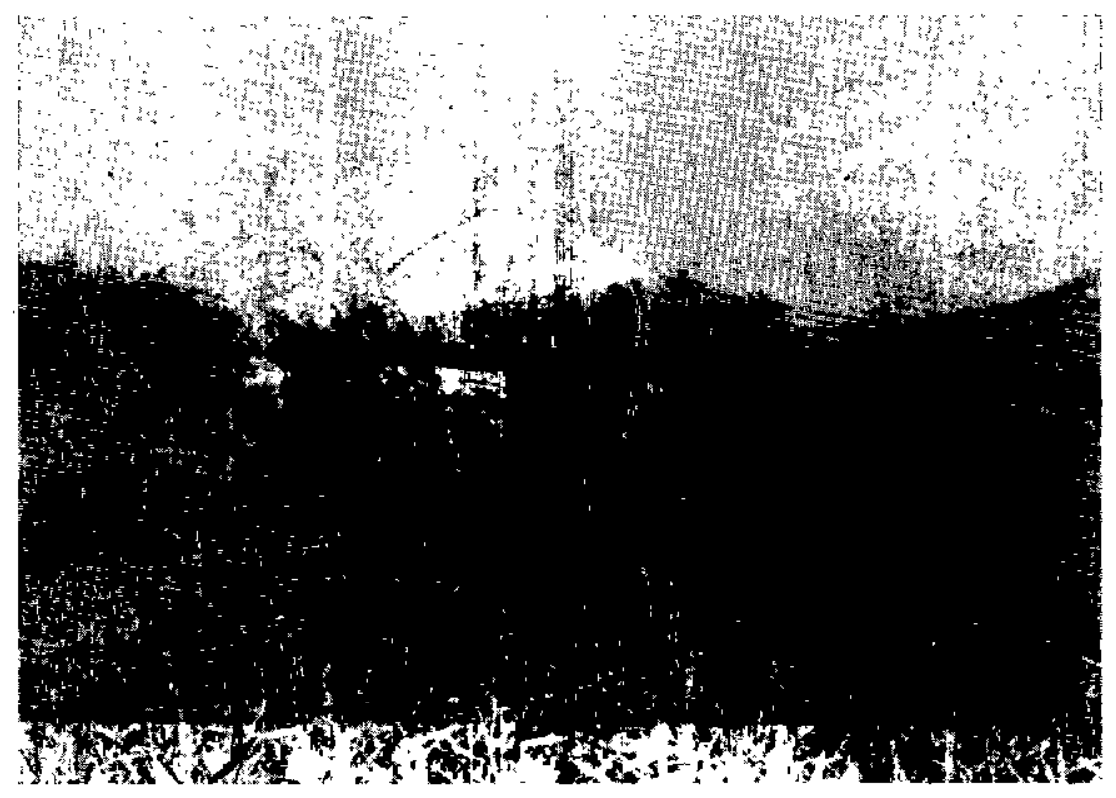

No 2. - Sporobolus pyramidalis touffe non broulée après 3 mois de pluies. 


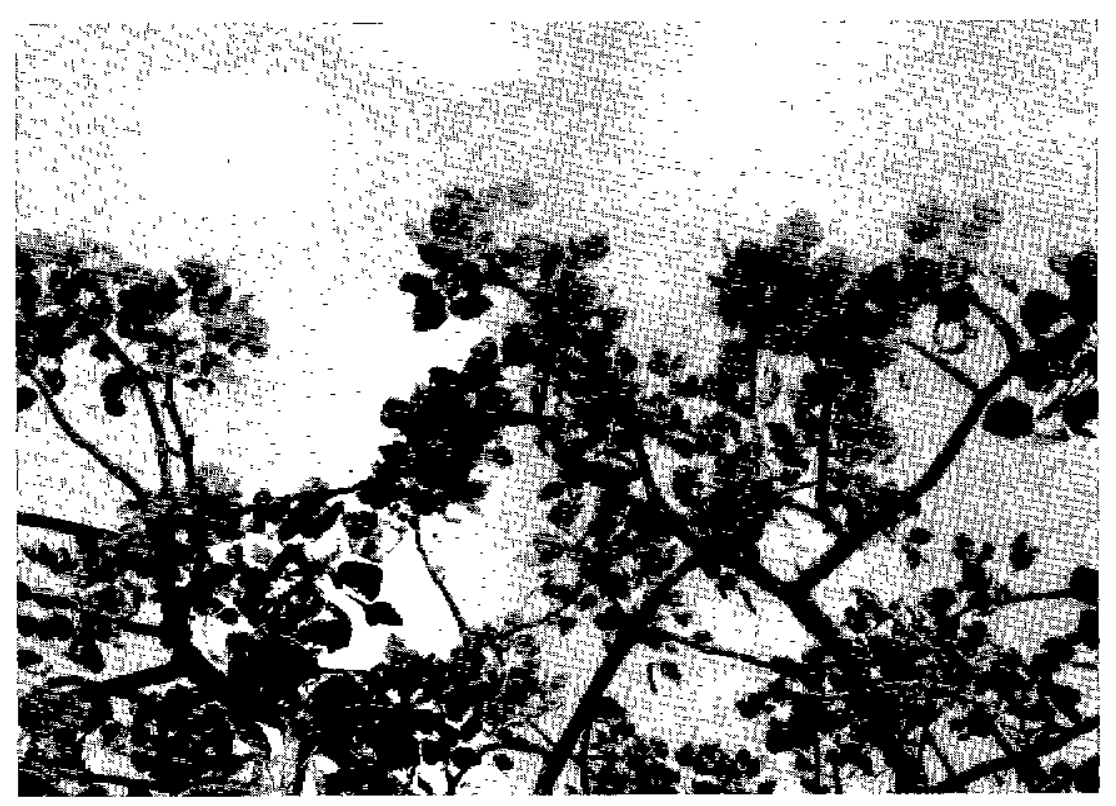

No 3. - Erythrina sigmoidea.

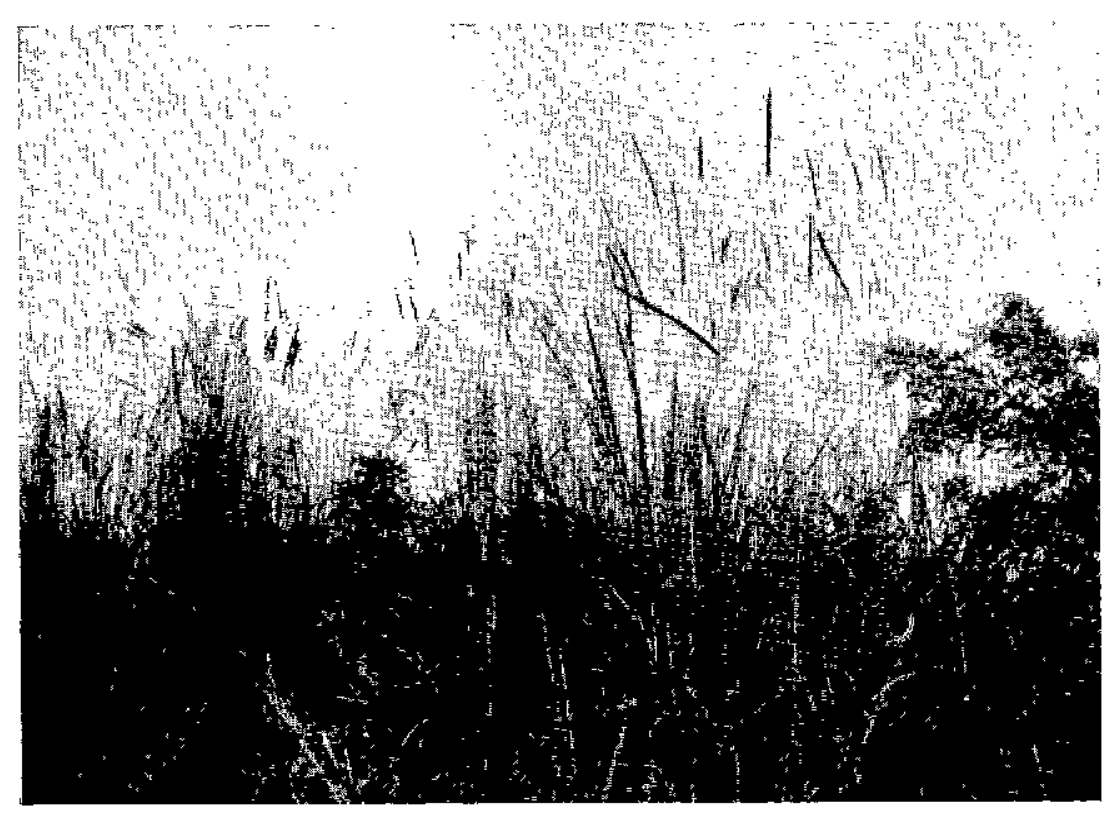

No 4. - Setaria sphacelata petite variété en fin de floraison (mi-juin 65). 


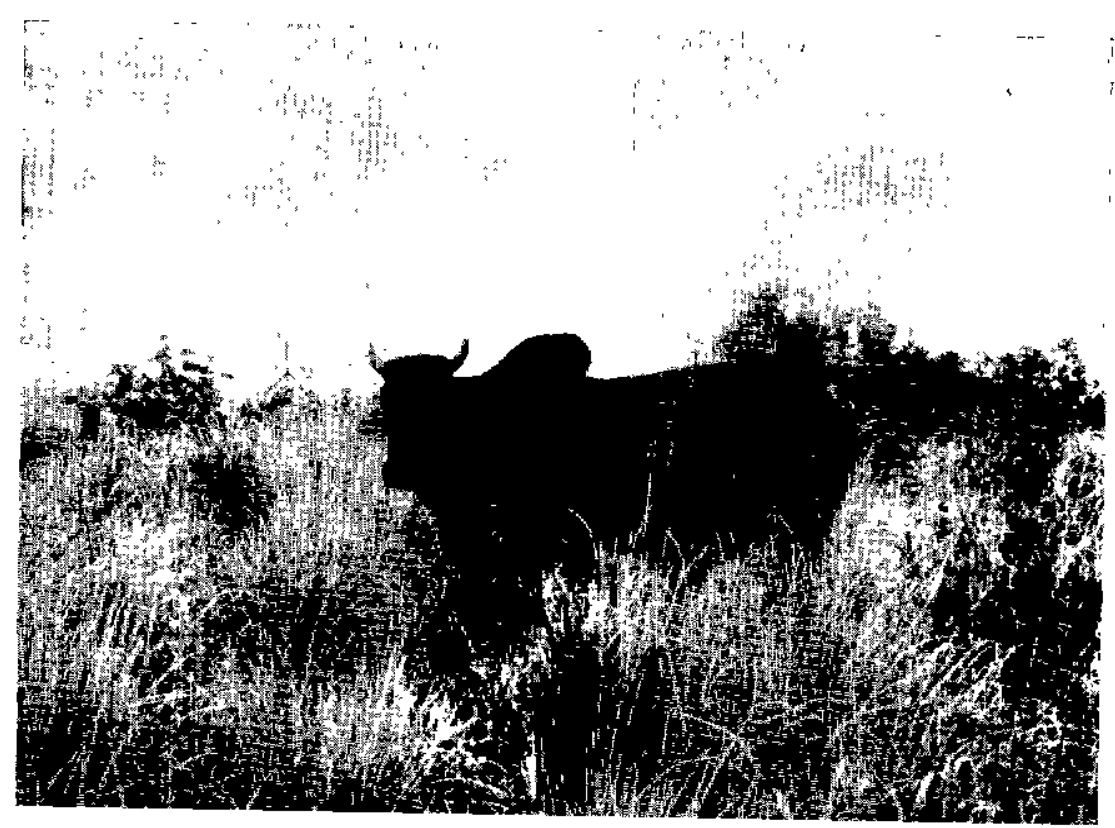

No 5. - Produits de croisement Brahman du Texas $\times$ Zébu pulfuli au pâturage.

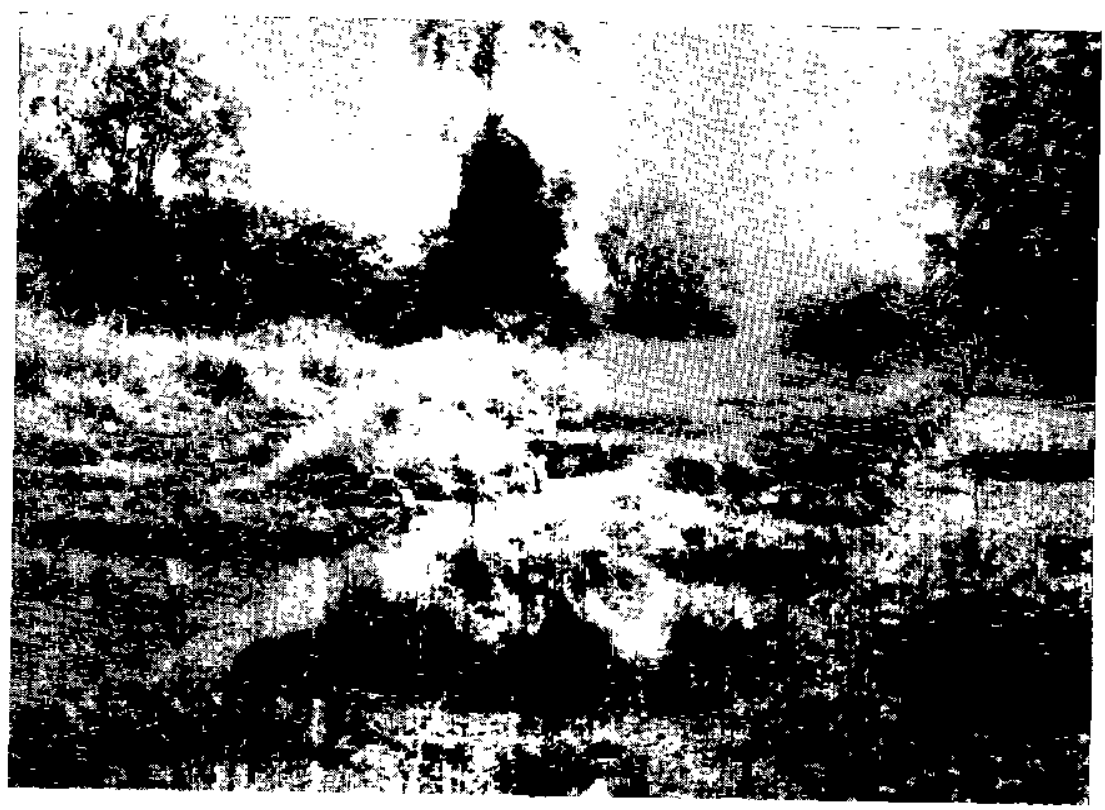

No 6. - Le jardin botanique en cours de réaménagement. 


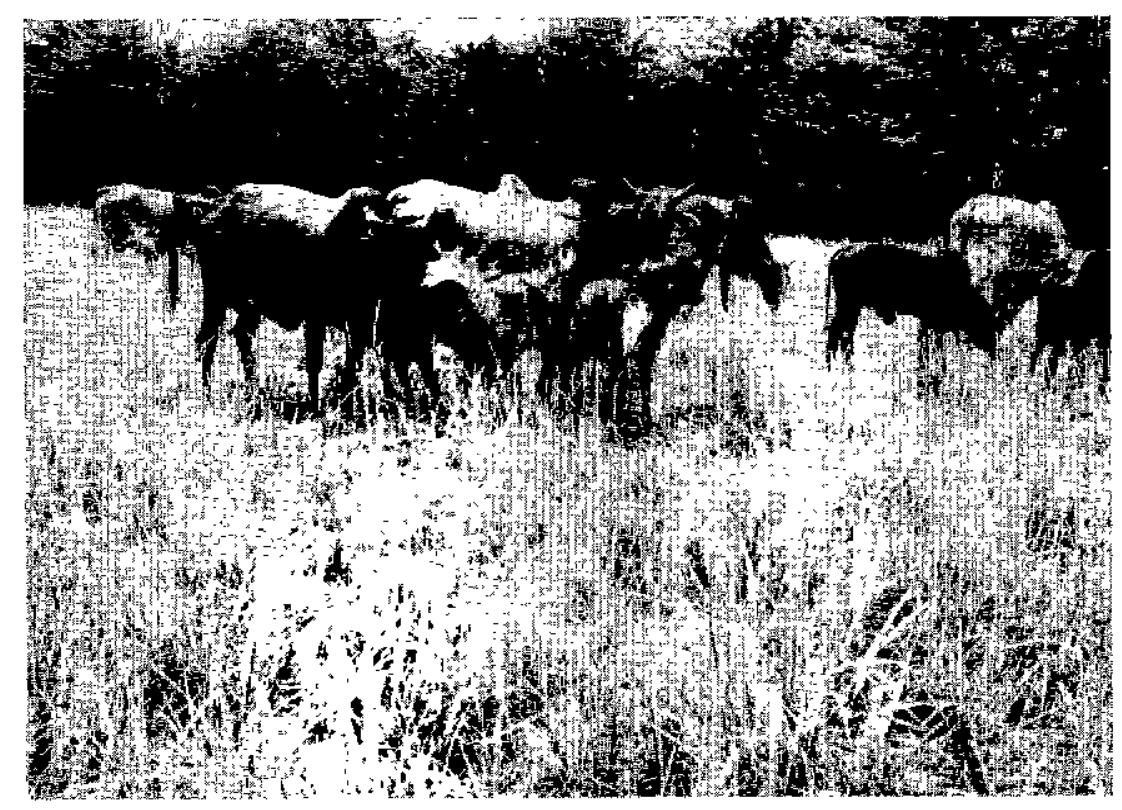

No 7. - Vaches pulfuli et leurs veaux à sang Brohman

au pâturage dans d'anciennes parcelles d'essais.

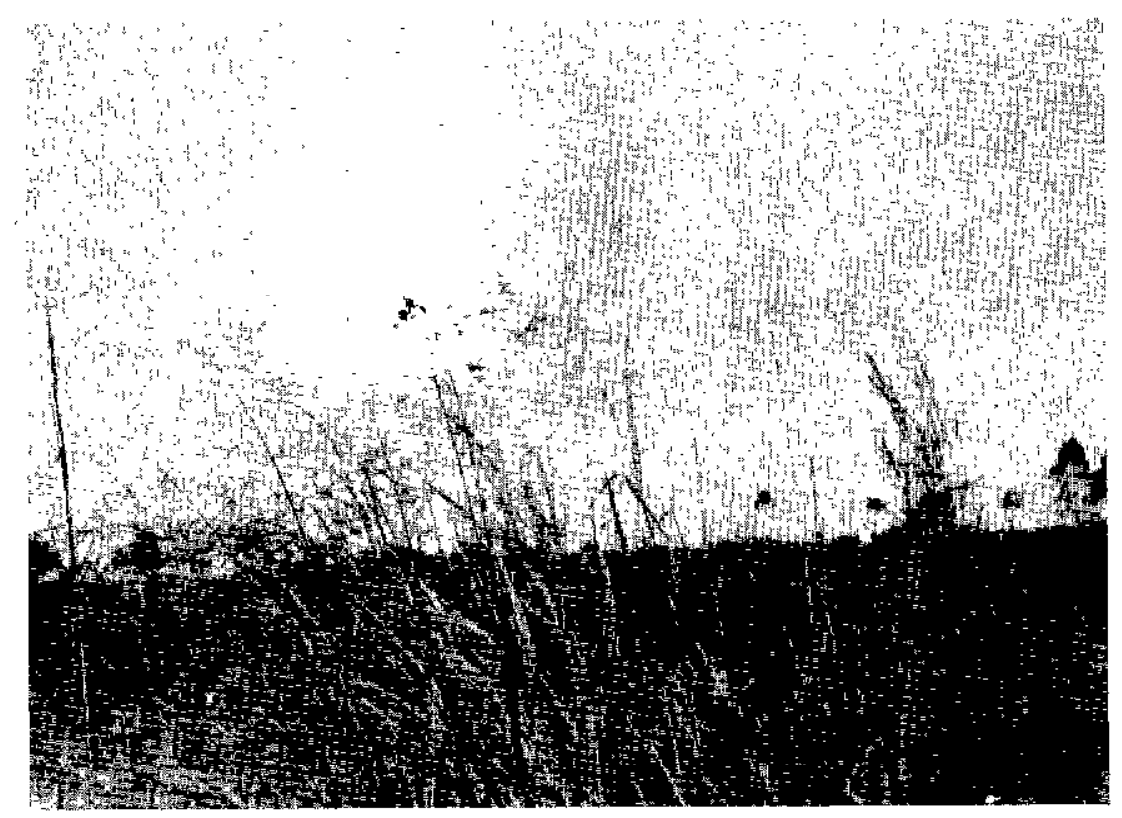

No 8. - La pousse de Panicum phrogmitoides prend vite les animaux de vitesse en début de saison des plures. 


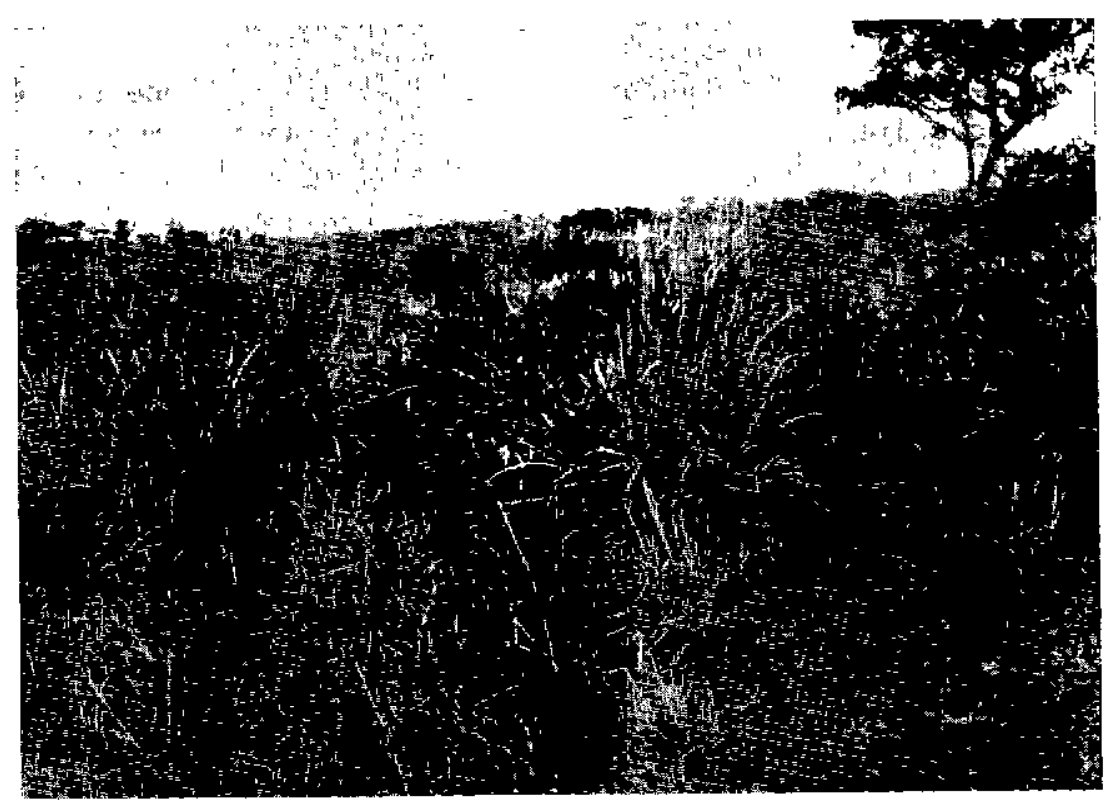

No 9. - Dans des conditions de charge insuffisante (ici $250 \mathrm{~kg}$ à l'hectare en début de saison des pluies). Le Ponicum phragmitoides constitue des refus importants.

\section{SUMMARY}

\section{Grazing study in the Cameroonian Adamawa}

The Adamawa plateou, with its favourable climatic and sanitary conditions is a range cattle breeding area which will soon be overgrazed. The main reasons for overgrazing are a global increase of the herd, on one hand, and $a$ desertion of traditional pastures either already out of use through excessive grazing or known for their heavy glossina infestation on the other hand .

The subject is a study of the evolution of basic pastures under cericin ways of exploitation. Remedies or improvement techniques are proposed in some cases.

A farming method based on pasture rotation during the rainy season and the dry season is recommended.

\section{RESUMEN}

Esiudios de los pastos en Adamaua cameronés

La meseta del Adamaua con sus condiciones climáticas y sanitarias favorables es una región de cria extensiva sobrecargandose. Los principales motivos de la sobrecarga son el crecimiento global de las reses y el abandono de zonas de pastos esquilmadas por un recorrido abusivo o reputadas infestadas por las glosinas.

Asi se estudic en este documento la evolución de los pastos de base bajo la acción de ciertos modos de explotación. Se proponen remedios o técnicas de mejoramiento en ciertos casos. Al fin, se preconiza un modo de explotación a partir de la rotación de los pastos durante la estación de las lluvias y la estación seca. 


\section{BIBLIOGRAPHIE}

AUBREVILLE (A.). - Climats, forêts et désertification de l'Afrique Tropicale Paris, Soc. Ed. Geogr. Mar, et Col., 1950.

- Flore forestière Soudano-Guinéenne. Paris, Soc. Ed. Geogr. Mar. et Col. 1950. - La flore forestière de la Côte-d'lvoire (Publication no 15 du CTFT 1959).

FROMENT (D.). - Aménagement ef exploitation des pâturages à dominance de «Hyparrhenia » de la région de Nioka. Bull. Inf. I. N. E. A. C., 1960, 9 (1) : 49.

HUTCHINSON (J.), DALZIEL (J.M.). - Flora of west Tropical Africa. Millbank, London, Crown agents for oversed governments and administrations, 1954-1963.

JACQUES-FELIX (H.). - Les graminées d'Afrique Tropicale. Paris, I. R. A. T., 1962.
KOECHLIN (J.). - Etude sur les pâturages ef les questions fourragères en République Centrafricaine. Rev. élev. Méd. vét. Pays trop. 1962, 15 (1) : 43-73.

MEREDITH (D.) et Coll. - The Grasses and pastures of South Africa. Central news Agency, 1955.

MONNIER (F.) et PIOT (J.). - Problèmes de pâturage dans l'Adamaoua. Bois Forêts trop. $1964(97): 3-15 ;(98): 13-25$.

ROBYNS (W.). - Flore Agrostologique du Congo Belge et du Ruanda Urundi - II Panicées. Bruxelles, Imp. Goemaere, 1934. RINEY (T.). - Principe et application d'une méthode de terrain rapide pour décrìre l'état actuel ef les tendances évolutives de la conservation dans les régions pastorales semi-arides. Sols afr., 1963, 8(2) : 259-334 